\title{
Positive airway pressure treatment for opioid-related central sleep apnea, where are we now?
}

\author{
David Wang • Luke Rowsell • Brendon J. Yee
}

Received: 2 October 2013 / Accepted: 4 October 2013 / Published online: 26 October 2013

(C) Springer-Verlag Berlin Heidelberg 2013

There are five subtypes of central sleep apnea (CSA) syndromes in adults: CSA due to Cheyne-Stokes breathing, high altitude-related periodic breathing, CSA due to medical condition not Cheyne-Stokes, CSA due to drug or substance, and primary (idiopathic) CSA [1,2]. The CSA due to drug or substance primarily refers to opioid-related CSA, which has been rarely studied $[2,3]$. Opioid-related CSA has only been recognized approximately since 2000 [3-7]. The pathogenesis is unclear and unlikely to be explained by one single mechanism [3, 7]. Two potential mechanisms have been postulated $[7,8]$. From a ventilatory control aspect, chronic opioid users have a depressed ventilatory response to hypercapnia but an augmented response to hypoxia [7, 9]. The imbalance in ventilatory chemoreflexes may contribute to breathing instability. This mechanism can be partially seen in CSA related to high altitude, heart failure and hypercapnic respiratory failure $[3,7,9]$. From a CNS aspect, opioids may selectively inhibit the inspiratory rhythm generator in the preBötzinger complex but not the expiratory rhythm generator in the retrotrapezoid nucleus/parafacial respiratory group. This potential imbalance in the respiratory rhythm generators may also contribute to the CSA and ataxic breathing [8].

Treatments of opioid-related CSA have been rarely reported. There are only six studies in the field, and all of them

\section{Wang $(\bowtie) \cdot$ B. J. Yee}

Department of Respiratory and Sleep Medicine, Royal Prince Alfred

Hospital, Missenden Rd, Camperdown, New South Wales 2050,

Australia

e-mail: david.wang@sydney.edu.au

D. Wang $\cdot$ L. Rowsell $\cdot$ B. J. Yee

Woolcock Institute of Medical Research, University of Sydney,

Glebe Point Rd, Glebe, New South Wales, Australia

L. Rowsell • B. J. Yee

Centre for Integrated Research and Understanding of Sleep (CIRUS),

University of Sydney, Sydney, New South Wales, Australia are retrospective observations or case reports [10-15]. While CSA in opioid users is related to opioid blood concentration/ dose $[6,7]$, reducing the dose of opioids may not be an easy option with concerns about uncontrolled pain and/or increased drug seeking behavior [16]. The earliest report for opioidrelated CSA treatment using positive airway pressure (PAP) is from 2007 [15]. Thirteen opioid users (five with CSA, eight with complex sleep apnea) were among an overall group of $100 \mathrm{CSA} /$ complex sleep apnea patients who received adaptive servoventilation (ASV) treatment. Median apnea hypopnea index (AHI) of the overall group dropped from 48 per hour at baseline to 5 per hour. However, the breakdown analysis of the 13 opioid users was not provided [15]. In 2008, PAP treatment for opioid-related CSA was reported in five chronic opioid users [12]. With standard CPAP therapy, CSA in the five patients worsened from 26 to 37 per hour. The authors then applied ASV which successfully eliminated CSA [12]. Interestingly, in the same issue of the journal, Farney et al. reported a similar retrospective analysis of CSA treatment in 22 chronic opioid users using ASV. In their study, the decrease of the central apnea index (CAI) was not significant (from 26.4 to 15.6 per hour), and no improvement was found in ataxic breathing and overnight oxygenation [11]. In 2009, another case report showed that Bi-level PAP (with a backup rate of 12-16 breath per minute) treatment in four chronic opioid users significantly reduced AHI from 60.2 to 16.6 per hour. CSA was eliminated in three out of the four CPAP nonresponders [14]. In 2012, two larger retrospective CSA treatment studies were reported [10, 13]. Ramar et al. reported a success rate (overall $\mathrm{AHI}<10$ ) of $59.6 \%$ (28 out of 47 ) for opioid-related CSA treatment using ASV. The success rate was similar $(61.7 \%, 29$ out of 47$)$ using a criteria of AHI $<5$ under optimal ASV end-expiratory pressure [13]. In the other retrospective study, 47 chronic opioid users were treated with $\mathrm{CPAP}, \mathrm{CPAP}+\mathrm{O}_{2}$, or $\mathrm{BiPAP}+\mathrm{O}_{2}$ in a stepwise order until optimal titration reached. Forty one patients completed the 
treatment protocol with optimal response achieved in 36 (89 \%) patients. CSA was eliminated in 54,28 , and $10 \%$, respectively, of cases with the three treatments. These treatments produced a significant decline in the AHI, CAI, and arousal index, and improved oxygenation [10].

The above six studies provide evidence for PAP treatment for opioid-related CSA. The discrepancy between the results may be related to the differential definitions of CSA, methodologies/settings of PAP, the dose of opioids, and the underlying medical conditions. Due to the low level of evidence, the recent published AASM Practice Parameters has not given a recommendation for the treatment of opioidrelated CSA [2]. Further studies were suggested to compare the efficacy of CPAP, BiPAP, and ASV in the treatment of opioidrelated CSA. Meanwhile, "assessment for discontinuation of opioid use and substitution of other forms of pain relief seems prudent" [2].

Given the above context, the retrospective study conducted by Troitino and colleagues in this issue of the journal provides further evidence in the field [17]. They compared the treatment outcome and adherence rate of CPAP, BiPAP, and ASV in 34 opioid-related CSA (O-CSA) and 61 idiopathic CSA (I-CSA) patients [17]. CSA was defined as CAI $>5$ per hour and $\mathrm{CAI} \geq 50 \%$ of the AHI. PAP compliance was defined as time spent on PAP $>4$ per hour per night on $70 \%$ of nights. Only those with chronic opioid use $>6$ months were included for the O-CSA subtype. CSA patients with other major medical conditions were excluded for the idiopathic subtype. Interestingly, both groups had substantial baseline hypoxemia. The I-CSA group had a high \%sleep time with $\mathrm{SpO}_{2}<90 \%$ (\%T90) of $37 \%$ and $\mathrm{Mean}_{\mathrm{SpO}}$ of $91 \%$ and $\mathrm{SpO}_{2}$ nadir of $81 \%$. This is somewhat different to our normal understanding of idiopathic CSA, which belongs to a type of hypocapnic CSA, with low $\mathrm{PCO}_{2}$ and hyperventilation, and not associated with major hypoxemia $[18,19]$. Nevertheless, the high arousal index in this group (38 per hour) seems in line with the classic features of I-CSA [19]. The O-CSA group had prominent CSA events with an average CAI of 29.3 pre hour, together with as a high prevalence of ataxic breathing (32\%), \%T90 $(41.8 \%)$ and a low $\mathrm{SpO}_{2}$ nadir of $81.7 \%$ [17]. Following the stepwise treatment algorithm of CPAP, BiPAP and ASV (if previous treatment failed), both CSA groups dropped their mean $\mathrm{AHI}$ to $\sim 5$ per hour, $\% \mathrm{~T} 90$ to $\sim 5 \%$ and $\mathrm{SpO}_{2}$ nadir increased to $\sim 88 \%$. Arousal index in the I-CSA group dropped by $\sim$ half. The possible mechanism of the reduced CAI and arousal index with PAPs may relate to a reduced loop gain and therefore a reduced hyperventilation and cyclic periodic breathing pattern [20]. CPAP, BiPAP, and ASV were comparable in eliminating CSA and improving oxygenations in both groups of CSA. The initial CPAP compliance was $24 \%$ for O-CSA group and $38 \%$ for I-CSA group. Twelve months PAP adherence rate of both O-CSA and I-CSA groups were similarly low $(\sim 20 \%)$ [17].
There are some useful discussion points from the Troitino et al. study, and other six studies involving PAP treatment of opioid-related CSA:

1. It seems that carefully titrated PAP treatments can improve oxygenation and reduce CSA events in the majority of the opioid-related CSA patients, although low PAP adherence is clearly a problem. Opioids are recognized as potent respiratory depressants. Hypoxia and hypercapnia can be major features in some opioidrelated CSA patients, and it would certainly be beneficial if these abnormalities can be ameliorated.

2. CSA in chronic opioid users has different presentations with some patients not having impaired oxygenation during sleep [7]. For those with significant CSA but normal oxygenation, it could be controversial to use PAP just to reduce the CSA events. Importantly, we do not have firm evidence that opioid-related CSA itself, without concomitant hypoxemia, can lead to increased mortality or significant cardiovascular or neurological consequences. In our previous studies, we found that chronic opioid users have increased daytime sleepiness and reduced daytime function, but CSA did not play a role in those changes [21]. In terms of mortality, PAP could be more important during the opioid induction phase as nearly half of the opioid-related deaths occur during the first 2 weeks of that acute induction period [22]. Without concomitant hypoxia/hypercapnia, it would be even more controversial to discontinue/significantly reduce the opioid use just to avoid CSA, as the trade-off is usually increased pain or increased drug seeking behavior [16].

3. Both ASV and BiPAP are much more expensive than CPAP but do not show vastly superior outcomes, a practical approach could be still using CPAP as the initial mode of treatment. ASV and BiPAP could be considered as alternative modalities if CPAP fails.

4. Given the different presentations of opioid-related CSA [7] and high inter-individual variability in opioid responses [23], a personalized medicine tailoring treatments according to individual features (like overnight hypoxemia, hypercapnia, CSA, awake ventilatory responses, daytime sleepiness/alertness, etc.) could be a more sensible approach.

5. Proper designed RCT studies with larger sample size are needed to compare the clinical and neurological outcomes from CPAP (and other modalities) treatment and standard care. More in-detail respiratory analyses (further to CSA and ataxic breathing) and neurocognitive function tests (such as EEG spectral analyses) could be considered. In addition, long-term data are needed to explore any potential role of opioid-related breathing abnormalities (such as CSA and ataxic breathing) in mortality and cardiovascular and neurocognitive consequences. 
Acknowledgments We would like to thanks Prof Ron Grunstein, Dr Amanda J Piper, and Dr Peter Buchanan for providing valuable comments. Dr David Wang is supported by NHMRC Health Professional Research Fellowship and Sydney Medical School Early Career Researcher/New Staff Award. Luke Rowsell is supported by CIRUS.

\section{References}

1. American Academy of Sleep Medicine (2005) International classification of sleep disorders, second edition: diagnostic and coding manual. American Academy of Sleep Medicine, Westcherter

2. Aurora RN, Chowdhuri S, Ramar K, Bista SR, Casey KR, Lamm CI, Kristo DA, Mallea JM, Rowley JA, Zak RS, Tracy SL (2012) The treatment of central sleep apnea syndromes in adults: practice parameters with an evidence-based literature review and metaanalyses. Sleep 35(1):17-40. doi:10.5665/sleep. 1580

3. Wang D, Teichtahl H (2007) Opioids, sleep architecture and sleepdisordered breathing. Sleep Med Rev 11(1):35-46

4. Farney RJ, Walker JM, Cloward TV, Rhondeau S (2003) Sleepdisordered breathing associated with long-term opioid therapy. Chest 123(2):632-639

5. Teichtahl H, Prodromidis A, Miller B, Cherry G, Kronborg I (2001) Sleep-disordered breathing in stable methadone programme patients: a pilot study. Addiction 96(3):395-403

6. Walker JM, Farney RJ, Rhondeau SM, Boyle KM, Valentine K, Cloward TV, Shilling KC (2007) Chronic opioid use is a risk factor for the development of central sleep apnea and ataxic breathing. J Clin Sleep Med 3(5):455-461

7. Wang D, Teichtahl H, Drummer OH, Goodman C, Cherry G, Cunnington D, Kronborg I (2005) Central sleep apnea in stable methadone maintenance treatment patients. Chest 128:1348-1356

8. Feldman JL, Del Negro CA (2006) Looking for inspiration: new perspectives on respiratory rhythm. Nat Rev Neurosci 7(3):232-242

9. Teichtahl H, Wang D, Cunnington D, Quinnell T, Tran H, Kronborg I, Drummer OH (2005) Ventilatory response to hypoxia and hypercapnia in stable methadone maintenance treatment patients. Chest 128:1339-1347

10. Chowdhuri S, Ghabsha A, Sinha P, Kadri M, Narula S, Badr MS (2012) Treatment of central sleep apnea in U.S. veterans. J Clin Sleep Med 8(5):555-563. doi:10.5664/jcsm.2156

11. Farney RJ, Walker JM, Boyle KM, Cloward TV, Shilling KC (2008) Adaptive servoventilation (ASV) in patients with sleep disordered breathing associated with chronic opioid medications for nonmalignant pain. J Clin Sleep Med 4(4):311-319
12. Javaheri S, Malik A, Smith J, Chung E (2008) Adaptive pressure support servoventilation: a novel treatment for sleep apnea associated with use of opioids. J Clin Sleep Med 4(4):305-310

13. Ramar K, Ramar P, Morgenthaler TI (2012) Adaptive servoventilation in patients with central or complex sleep apnea related to chronic opioid use and congestive heart failure. J Clin Sleep Med 8(5):569576. doi: $10.5664 /$ jcsm. 2160

14. Alattar MA, Scharf SM (2009) Opioid-associated central sleep apnea: a case series. Sleep Breath 13(2):201-206. doi:10.1007/ s11325-008-0221-7

15. Allam JS, Olson EJ, Gay PC, Morgenthaler TI (2007) Efficacy of adaptive servoventilation in treatment of complex and central sleep apnea syndromes. Chest 132(6):1839-1846. doi:10.1378/chest.071715

16. American Pain Society (1997) The use of opioids for the treatment of chronic pain. A consensus statement from the American Academy of Pain Medicine and the American Pain Society. Clin J Pain 13(1):6-8

17. Troitino A, Labedi N, Kufel T, El-Solh AA (2013) Positive airway pressure therapy in patients with opioid-related central sleep apnea. Sleep Breath. doi:10.1007/s11325-013-0894-4

18. Xie A, Rutherford R, Rankin F, Wong B, Bradley TD (1995) Hypocapnia and increased ventilatory responsiveness in patients with idiopathic central sleep apnea. Am J Respir Crit Care Med 152(6 Pt 1):1950-1955. doi:10.1164/ajrccm.152.6.8520761

19. Xie A, Wong B, Phillipson EA, Slutsky AS, Bradley TD (1994) Interaction of hyperventilation and arousal in the pathogenesis of idiopathic central sleep apnea. Am J Respir Crit Care Med 150(2): 489-495. doi:10.1164/ajrccm.150.2.8049835

20. Edwards BA, Sands SA, Feeney C, Skuza EM, Brodecky V, Wilkinson MH, Berger PJ (2009) Continuous positive airway pressure reduces loop gain and resolves periodic central apneas in the lamb. Respir Physiol Neurobiol 168(3):239-249. doi:10.1016/j. resp.2009.07.006

21. Wang D, Teichtahl H, Goodman C, Drummer O, Grunstein RR, Kronborg I (2008) Subjective daytime sleepiness and daytime function in patients on stable methadone maintenance treatment: possible mechanisms. J Clin Sleep Med 4(6):557-562

22. Caplehorn JR, Drummer OH (1999) Mortality associated with New South Wales methadone programs in 1994: lives lost and saved. Med J Aust 170(3):104-109

23. Wang D, Somogyi AA, Yee BJ, Wong KK, Kaur J, Wrigley PJ, Grunstein RR (2013) The effects of a single mild dose of morphine on chemoreflexes and breathing in obstructive sleep apnea. Respir Physiol Neurobiol 185(3):526-532. doi:10.1016/j. resp.2012.11.014 